\title{
Self-organization patterns in electroluminescence of bistable ZnS:Mn thin-film structures
}

\author{
N.A. Vlasenko*, H.-G. Purwins**, Z.L. Denisova*, Ya.F. Kononets*, \\ F.-J. Niedernostheide**, L.I. Veligura*, S. Zuccaro** \\ ${ }^{*} V$. Lashkaryov Institute of Semiconductor Physics, NAS of Ukraine, 41, prospect Nauky, 03028 Kyiv, Ukraine \\ Phone: +380 (44) 2656252, E-mail:vlasenko@isp.kiev.ua. \\ *Institute of Applied Physics, University of Muenster, Corrensstrasse 2/4, Muenster, D-48149, Germany, \\ Phone:00492518333511,E-mail:purwins@uni-muenster.de
}

\begin{abstract}
An overview of recent original results concerning self-organized pattern formation in the emission of bistable alternating current $\mathrm{ZnS}: \mathrm{Mn}$ thin - film electroluminescent structures (TFELS) as a dissipative system is given. The influence of technological factors, affecting the properties TFELS, and driving conditions on both the patterns and the hysteresis of the charge- voltage dependence that is responsible for bistability of the TFELS are considered. The correlation between patterns and the shape of the hysteresis is analyzed. The physical processes with positive and negative feedback, which serve, respectively, as activator and inhibitor in the given dissipative system, are discussed.
\end{abstract}

Keywords: self-organization, patterns, electroluminescence, $\mathrm{ZnS}: \mathrm{Mn}$, thin-film structures, bistability.

Paper received 28.11.03; accepted for publication 30.03.04.

Spontaneous formation of spatially inhomogeneous dynamic states in dissipative systems is extensively studied since the 1970s in chemistry, biophysics, physics of plasma and semiconductors, and other fields [1-3]. Solidstate electronic dissipative systems showing light-emitting spatio-temporal patterns, are of essential interest in basic research and potential applications. Bistable $\mathrm{ZnS}: \mathrm{Mn}$ thin-film electroluminescent structures (TFELS) driven by an AC voltage are one of such systems [4-6]. Various patterns such as filaments, micro-domains and macro-domains, autosolitons, and different autowaves (annular, periodic, chaotic, "flip-flop") are generated in the emission of the TFELS having certain properties under suitable driving conditions (Fig. 1). First, some dynamic patterns in AC ZnS:Mn TFELS with a hysteretic luminance - voltage $(\mathrm{L}-\mathrm{V})$ characteristic have been reported in [7] They have been explained by a thermal model. According to this model, the flow of heat spreads from some primary switched - on areas. The heating results in the appearance of electroluminescence $(E L)$ in neighbouring areas due to a decrease of the threshold voltage $\left(V_{t h}\right)$ with increasing temperature $(T)$. This excitation wave is then followed by a quenching of EL when the temperature after switching - on exceeds some critical temperature, beyond which the hysteresis disappears. However, in the framework of this model, the variety of dynamic patterns observed in TFELS cannot be explained. In particular, the fact that the flip-flop autowaves (AWs) may appear without a marked heating of a driving cell [5] is not consistent with the thermal model. A study of TFELS, showing the patterns, as a dissipative system with specific activator and inhibitor processes was begun a decade ago [4-6, 8, 9]. Recently, detailed experimental investigations have been carried out [10-19] to ascertain which TFELS properties, technological factors, EL excitation conditions as well as physical processes are responsible for the appearance of various kinds of self-organized patterns and the bistability of the TFELS. In this paper, the main results of these investigations are summarized. As well, an identification of the activating and inhibiting mechanisms and a consistent explanation of observed self-organization phenomenon in bistable ZnS:Mn TFELS are given.

The TFELS under study consisted of insulator-semiconductor-insulator (I-S-I) layers sandwiched between two electrodes, namely, a transparent Indium Thin Oxide (ITO) film deposited on a glass substrate and an outer Al film. In the first type of the TFELS the $S$-layer ( $\mathrm{ZnS}: \mathrm{Mn})$ and the I layers $\left(\mathrm{SiO}_{2} / \mathrm{Al}_{2} \mathrm{O}_{3}\right)$ were deposited by electron-beam evaporation (EBE), whereas in the second type, the $\mathrm{ZnS}: \mathrm{Mn}$ film and the $\mathrm{Al}_{\mathrm{x}} \mathrm{Ti}_{\mathrm{y}} \mathrm{O}_{\mathrm{z}}$ insulator layers were grown by the atomic layer epitaxy (ALE) 


\section{N.A. Vlasenko et al.: Self-organization patterns in electroluminescence of bistable...}

technique with the use of chlorine precursors $\left(\mathrm{ZnCl}_{2}\right.$, $\mathrm{MnCl}_{2}$ ) and $\mathrm{H}_{2} \mathrm{~S}$ [20]. These types of TFELS differ in both the crystal structures of the $\mathrm{ZnS}: \mathrm{Mn}$ films and their electrical properties. ZnS:Mn EBE films are fine-grained with a submicron surface roughness [21]. In addition to Mn iso valent traps (IVT) $\left[\mathrm{Mn}_{\mathrm{Zn}}^{2+}\right]^{0}[11]$ they contain only intrinsic compensated defects ( e.g. zinc and sulfur vacancies, $\left.V_{\mathrm{Zn}}, V_{\mathrm{S}}\right)$. ZnS:Mn ALE films have a rather large-grain structure with a smooth crystallite surface [21]. They contain additional chlorine impurity that forms shallow donors $\mathrm{Cl}_{\mathrm{S}}^{+}$located $\sim 0.3 \mathrm{eV}$ below the $\mathrm{ZnS}$ conduction band [12].

These differences give obviously rise to the different kind of patterns observed in the emission of the EBE and ALE TFELS. All kinds of the above mentioned patterns arise in the former, but only flip-flop AWs are found in the latter. Moreover, flip-flop AWs qualitatively differ in the two types of TFELS. In the first type, they form irregularly-shaped domains whose edges change as the front propagates (Fig. 1, f). In the second type, these AWs represent circular domains whose diameter increases with time (Fig. $1, g$ ).

Self-organized patterns were observed only in bistable TFELS with the hysteretic $L(V)$ and charge-voltage ( $Q$ $V)$ characteristics. Therefore, main factors affecting both the hysteresis of the characteristics and the formation of patterns were studied. The relationship between these two phenomena was also investigated. To ascertain the role

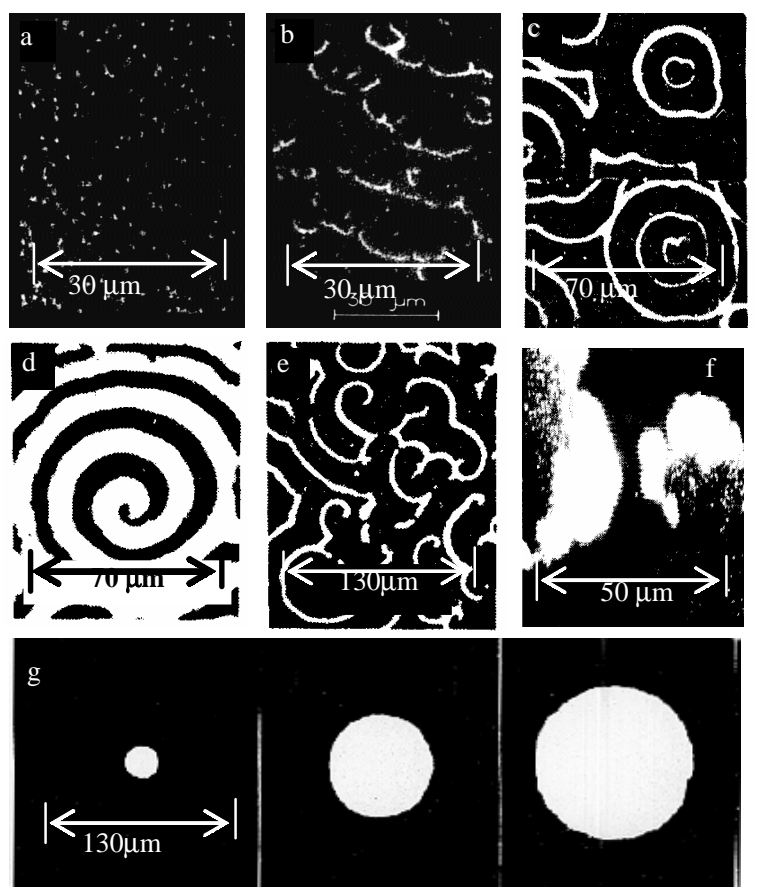

Fig. 1. Patterns observed in light emission of EBE samples $(a-f)$ and ALE samples $(g)$. (a) dynamic filaments; $(b-g)$ different autowaves: string (b), annular (c), spiral (d), chaotic (e), flip-flop irregular $(f)$ and circular $(g)$. Time between subsequent pictures in $(g)$ is $3 \mathrm{~s}$.

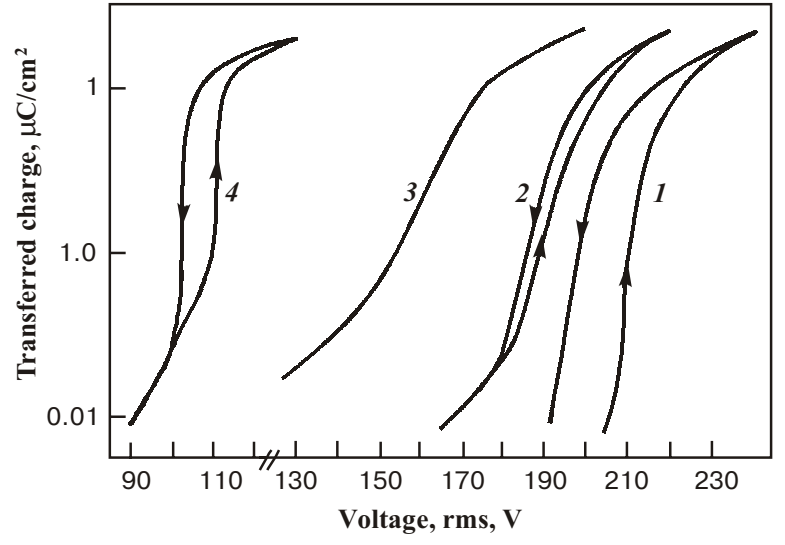

Fig. 2. Voltage dependence of transferred charge of EBE TFELS (1-3) and ALE TFELS (4) with different Mn concentration. $\mathrm{C}_{\mathrm{Mn}}$, at. \%: (1) - 3; (2) - 1.2; (3) - 0.5; (4) - 2. EBE TFELS were deposited at substrate temperature of $170^{\circ} \mathrm{C}$ and were annealed at $450^{\circ} \mathrm{C}$ in vacuum.

of different defects in the $\mathrm{ZnS}$ :Mn film, the following characteristics were studied:

- photodepolarization (PDP) spectra.

- the influence of the defects on $L(V)$ and $Q(V)$ characteristics.

- the dependencies of parameters of the hysteresis of the characteristics on the temperature and the frequency $(f)$ of the applied voltage $[15,16]$.

The hysteresis of the $L(V)$ and $Q(V)$ characteristics of TFELS results from the $S$-type instability of the latter. This instability is due to the positive feed back (PFB) between the tunnel emission and impact generation of free electrons and the enhancement of the field in the $\mathrm{ZnS}: \mathrm{Mn}$ film due to the formation of a polarization charge, in particular, a positive space charge (PSC). It has been confirmed $[10,15,16]$ that the hysteresis appears only at a rather high $\mathrm{Mn}$ concentration $\left(C_{\mathrm{Mn}}>\right.$ $\left.>10^{20} \mathrm{~cm}^{-3}\right)$. The width of the hysteresis loop increases as $C_{\mathrm{Mn}}$ increases up to $8 \cdot 10^{20} \mathrm{~cm}^{-3}$. This is accompanied by an increase of the slope of the $L(V)$ and $Q(V)$ characteristics (Fig. 2, [15]) that means a rapider enhancement of the field strength with increasing $V$, i.e. the faster formation of PSC. In the PDP spectra of both types of TFELS, an impurity photocurrent peak at $3.4-3.45 \mathrm{eV}$ was found to intensify significantly when $C_{\mathrm{Mn}}$ is increased (Fig. $3[11,15,16])$. This peak has been attributed to the photoionization of $\left[\mathrm{Mn}_{\mathrm{Zn}}^{2+}\right]^{0}$ IVTs created when $\mathrm{Mn}^{2+}$ ions substitute for $\mathrm{Zn}^{2+}$ ions. These IVT $\mathrm{S}$ are mainly responsible for the formation of PSC in two ways: firstly, by the direct impact ionization of IVTs [16] and, secondly, by the effective capture of free holes created as a consequence of the lattice impact ionization..

It has been shown $[10,15,16]$ that at the optimal $C_{\mathrm{Mn}}$ the hysteresis is significantly affected by the technological conditions of the $\mathrm{ZnS}: \mathrm{Mn}$ film preparation, which influence on the number of other defects. As for the hysteresis, shallow intrinsic donors turned out to be the most 


\section{N.A. Vlasenko et al.: Self-organization patterns in electroluminescence of bistable...}

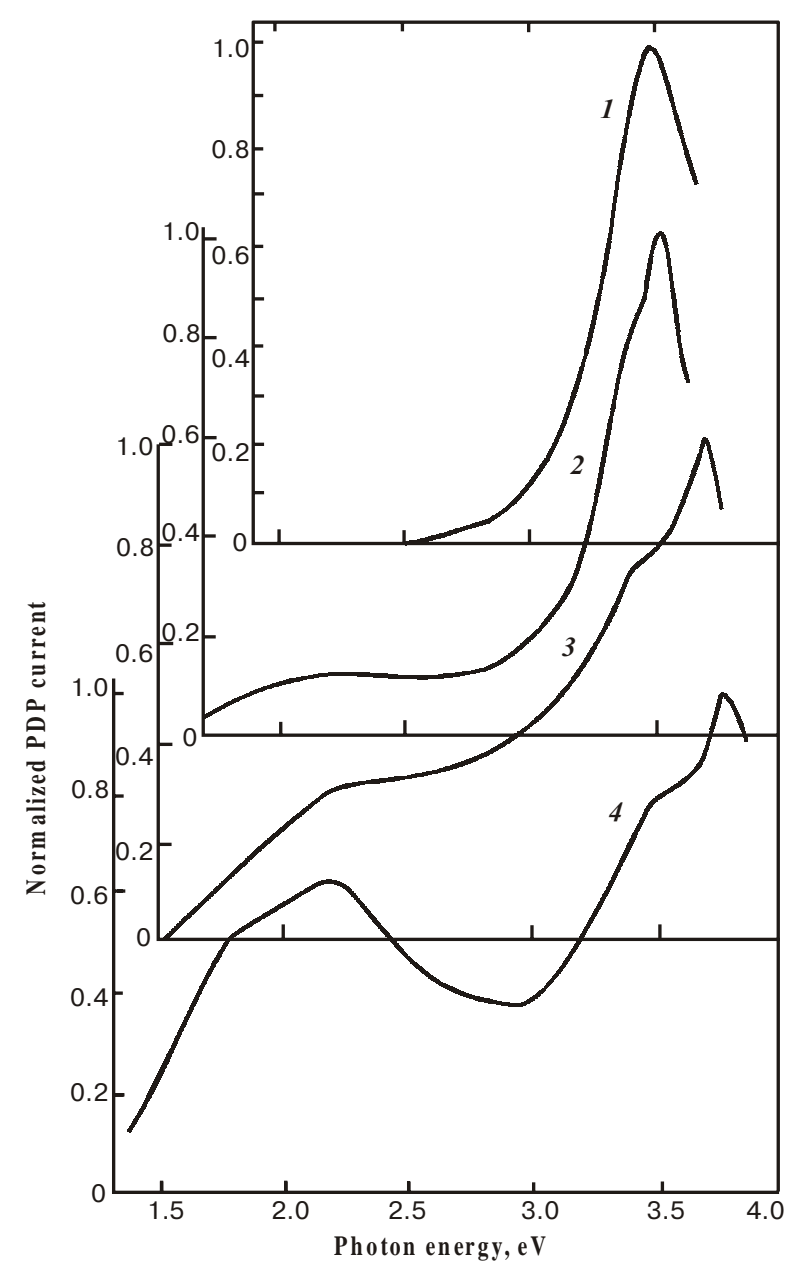

Fig. 3. PDP spectra of the same TFELS as in Fig. 2. Charging by DC voltage equal to $\sim V_{t h} / 2$ with " $+\mathrm{Al}$ " polarity. Illumination from the ITO side.

destructive defects. Thus, the deposition of the $\mathrm{ZnS}: \mathrm{Mn}$ EBE films at a low substrate temperature $\left(\leq 150^{\circ} \mathrm{C}\right)$ or annealing them at a high temperature $\left(\geq 500^{\circ} \mathrm{C}\right)$ in vacuum, causing a deterioration of the film stoichiometry, lead to a narrowing of the hysteresis loop. The presence of $\mathrm{Cl}$ in the $\mathrm{ZnS}: \mathrm{Mn}$ ALE films has a similar effect. The donors decrease the energy depth of the source of free electrons in TFELS. Electrons generated from such a source at a rather low field cannot be accelerated to the energy necessary for the impact ionization of both the deep centers and the lattice. Moreover, these electrons captured at the anodic interface reduce the field in the film due to the formation of the polarization charge. An analogous effect on the hysteresis also results from an increase in the leakage current in the $I$ layers [17]. It should be noted that the heavy Mn doping of the $\mathrm{ZnS}$ EBE films causes a significant decrease in the number of intrinsic defects because $\mathrm{Mn}^{2+}$ ions are incorporated into $\mathrm{Zn}$ vacancies. This is confirmed by two observations: 1) an increase in the threshold voltage and the disappearance of the initial weakly - increasing section of the $L(V)$ and $Q(V)$ characteristics at $V<V_{t h}$ (Fig. 2, [15]);2) a decrease in the impurity photocurrent in the PDP spectra related to single and double charged $\mathrm{Zn}$ vacancies, $\left(V_{\mathrm{Zn}}^{-}, V_{\mathrm{Zn}}^{2-}\right)$, and their complexes with intrinsic donors (Fig. 3, $[15,16])$. In ZnS:Mn, Cl ALE films these effects do not appear because the high concentration of donors requires a high concentration of $V_{\mathrm{Zn}}^{2-}$ acceptors to ensure charge neutrality. The presence of a high number of $V_{\mathrm{Zn}}^{2-}$ whose impact ionization probability is higher than that of $\operatorname{IVT}_{\mathrm{S}}$ [16], contributes to the formation of a part of PSC on these deep centers ( $\sim \mathrm{eV}$ above the valence band). This explains the observed differences in the temperature and frequency dependencies of the hysteresis loop parameters in the two types of TFELS [16].

As for the effect of various technological factors on the self-organized patterns, the high Mn concentration is also necessary for the appearance of such patterns in the emission of TFELS $[4,5,15]$. However, the too wide hysteresis loop is not favorable for the formation of dynamic patterns. Only static or "jumping" filaments are observed if the loop width $\left(\Delta V_{h}\right)$ exceeds $15 \mathrm{~V}$. Annular AWs and flip - flop AWs are found when $\Delta V_{h}$ is equal to $1-2 \mathrm{~V}$ and $5-10 \mathrm{~V}$, respectively $[4,5,15]$.

A narrow hysteresis loop is necessary for the formation of dynamic patterns owing to two reasons. Firstly, the differential negative resistance at the instable section of the $S$-shaped $Q(V)$ dependence is inversely proportional to $\Delta V_{h}$. For the existence of spontaneous oscillations, i.e. for the formation of AWs, this resistance has to be lower than the resistance of the electrodes and other resistive components of the electrical circuit connected in series with the TFELS. This requirement is more easily met if $\Delta V_{h}$ is small. Secondly, a narrow hysteresis loop at the optimal $\mathrm{C}_{\mathrm{Mn}}$ means that there are shallow electron states in the film bulk and at its interfaces. Shallow interface states are necessary for lateral spreading of a nonequilibrium charge initially generated in some switched - on areas. The following results confirm that this process, rather than the heat flow as suggested in [7], is mainly responsible for the propagation of an excitation wave along the film surface:

- The morphology of the ZnS:Mn film surface significantly affects the kind and the dynamics of patterns. This is well illustrated by the difference in flip-flop AWs in TFELS of the first and second types (Fig. 1,f,g.) with nearly the same Mn concentration and the same width of the hysteresis loop. Submicron heterogeneities cannot prevent the propagation of the heat flow, but they strongly affect the mechanism of charge spreading, since electrons pass through intercrystallite potential barriers at their local minima. The thermal model also cannot explain the different temperature at which flip-flop AWs arise in the first and second TFEL types $\left(60-80^{\circ} \mathrm{C}\right.$ and $\sim 20^{\circ} \mathrm{C}$, respectively). This is accounted for by the charge model, since the probability of passing of electrons through the intercrystallite barriers in a film with the rough surface increases with increasing temperature.

- There are observations of dynamic patterns, including AWs, in non-heated or weakly heated $\left(30-40^{\circ} \mathrm{C}\right)$ samples. So, flip-flop AWS in TFELS of the second type 


\section{N.A. Vlasenko et al.: Self-organization patterns in electroluminescence of bistable...}

occur without any marked heating of a driven cell. In some TFELS of the first type annealed at low temperatures $\left(330-350^{\circ} \mathrm{C}\right)$ in air after deposition of the upper I layer, annular AWs were observed at low-frequency driving $(2-5 \mathrm{~Hz})[18]$. In this case, the temperature at switched - on areas measured with liquid-crystal indicators does not exceed $40^{\circ} \mathrm{C}$.

- The temperature dependence of the propagation velocity $\left(v_{f}\right)$ of the AW front is essentially different in the two types of TFELS (Fig. 4). This cannot be explained by the thermal mechanism. In the charge model this difference can be attributed to the different energy depth $\Delta E$ of filled electron interface states in the $\mathrm{ZnS}: \mathrm{Mn} \mathrm{EBE}$ film containing only compensated intrinsic defects and in the ALE film having also shallow chlorine donors. In the former, the number of free electrons $\left(n_{e}\right)$ in the switched - on state, diffusion of which along the film surface results in lateral charge spreading, is low at room temperature. It increases exponentially with the rather high activation energy equal to $\Delta E$ with increasing $T . \Delta E$ ranges from $0.6 \mathrm{eV}$ to $0.7 \mathrm{eV}$ in $\mathrm{ZnS}$ :Mn EBE TFELS [13]. The diffusion of free electrons from switched - on areas along the film surface at the $I-S$ interfaces determines mainly the propagation velocity. This explains the observed exponential $v_{f}(T)$ dependence with the activation energy nearly equal to $0.65 \mathrm{eV}$. In the $\mathrm{ZnS}: \mathrm{Mn}, \mathrm{Cl} \mathrm{ALE}$ films, there is a considerable number of free electrons after switching - on even at room temperature. The relatively high $n_{e}$ results in the linear temperature dependence of the propagation velocity near this temperature. Such a dependence is characteristic for the free electron diffusion at constant $n_{e}$. With increasing temperature, $n_{e}$ increases exponentially. Moreover, in this case, the activation energy should be equal to the depth of $\mathrm{Cl}_{\mathrm{S}}^{+}$donors,

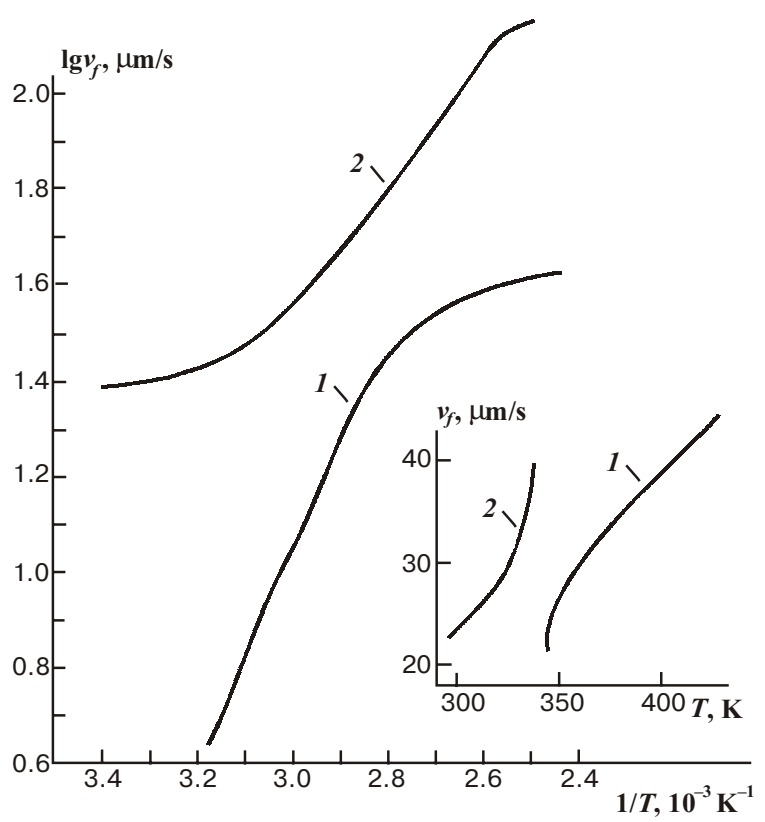

Fig. 4. Temperature dependence of front propagation velocity of annular AWs in EBE TFELS (1) and of flip-flop AWs in ALE TFELS (2). i.e. $\sim 0.3 \mathrm{eV}$. This is indeed confirmed by the measurements. The weaker $v_{f}(T)$ dependence at high temperatures $\left(>80^{\circ} \mathrm{C}\right)$ in both types of TFELS may be attributed to the depletion of the respective electron states.

The role of the $I$ layers in the formation of patterns was investigated as well [17]. These layers work as capacitive resistors limiting the avalanche breakdown in the case of the impact ionization inside the $S$ layer and preventing irreversible destruction. It has been suggested earlier [8,9] that the redistribution of the applied voltage between the $S$ layer and the $I$ layers during switching from the off to the on state is the main inhibitor, i.e. the process with the negative feed back (NFB), counteracting the activating processes which are tunneling and impact generation of free electrons. To confirm this suggestion, the influence of the $I$ layer thickness $\left(d_{i}\right)$ and the ratio $d_{i} / d_{s}$, where $d_{s}$ is thickness of the $S$ layer, on the patterns was studied. This ratio defines the magnitude of the coefficient $\alpha$ of the redistribution of the applied voltage, which is equal to $\left[1+\left(\varepsilon_{s} / \varepsilon_{i}\right)\left(d_{i} / d_{s}\right)\right]^{-1}$, where $\varepsilon_{s}$ and $\varepsilon_{i}$ are the permittivity of the $S$ and $I$ layers, respectively.

An increase of di in EBE TFELS made in one and the same technological cycle and showing only filaments as self-organized patterns results in an increase of the average distance between filaments and in the suppression of their cooperative behavior such as the formation of clusters and chains.

In the case of AW patterns, the influence of $d_{i}$ depends on the ratio $d_{i} / d_{s}$, i.e. on $\alpha$ (Fig. 5). Annular AWs with the narrow front were only observed when $\alpha$ was about $0.4-0.41$. An increase of $\alpha$ leads to a broadening of the AW front and, at even higher values of $\alpha$, to the transformation of AWs into micro-domains. For $\alpha>0.5$ nearly homogeneous emission was observed. A decrease of $\alpha$ leads to a diffuse filamentary structure of the AW front and, for even smaller values of $\alpha$, to the formation of macro-domains coexisting with a great number of filaments distributed almost homogeneously. The mobility of both the filaments and the domain walls decrease with decreasing $\alpha$. These results confirm the inhibitor role of the voltage drop across the $I$ layers. A reduction of the field in the $S$ layer owing to the voltage redistribution takes place not only in switched - on areas, but also in their vicinity because of bending of the equipotential lines in the $I$ layers.

The following conclusions about the activating and inhibiting processes in ZnS:Mn TFELS can be drawn from the results presented so far [22]. The activator may be divided into a local activator $\left(A_{l}\right)$ and a distributed one $\left(A_{d}\right)$. The former includes three main processes with the PFB (tunneling and impact generation of electrons, and formation of PSC), which are responsible for the $S$ shaped $Q(V)$ characteristic. The distributed activator causes the lateral propagation of the excitation wave. Non-equilibrium charge carries (electrons) spread along the $S-I$ interfaces from switched - on areas to neighboring areas under action of the tangential component of the electric field. This field, in turn, is created by the inhomogeneous charge distribution on the interface. The ac- 


\section{N.A. Vlasenko et al.: Self-organization patterns in electroluminescence of bistable...}
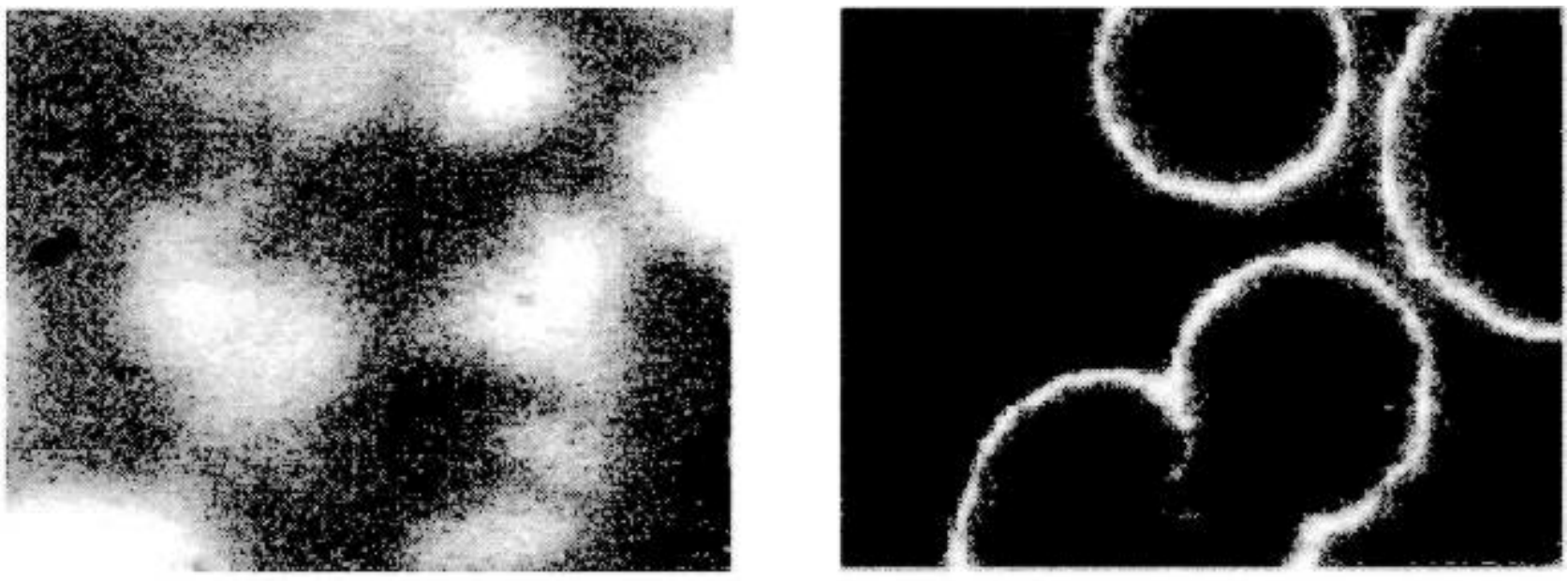

$\boldsymbol{a}$
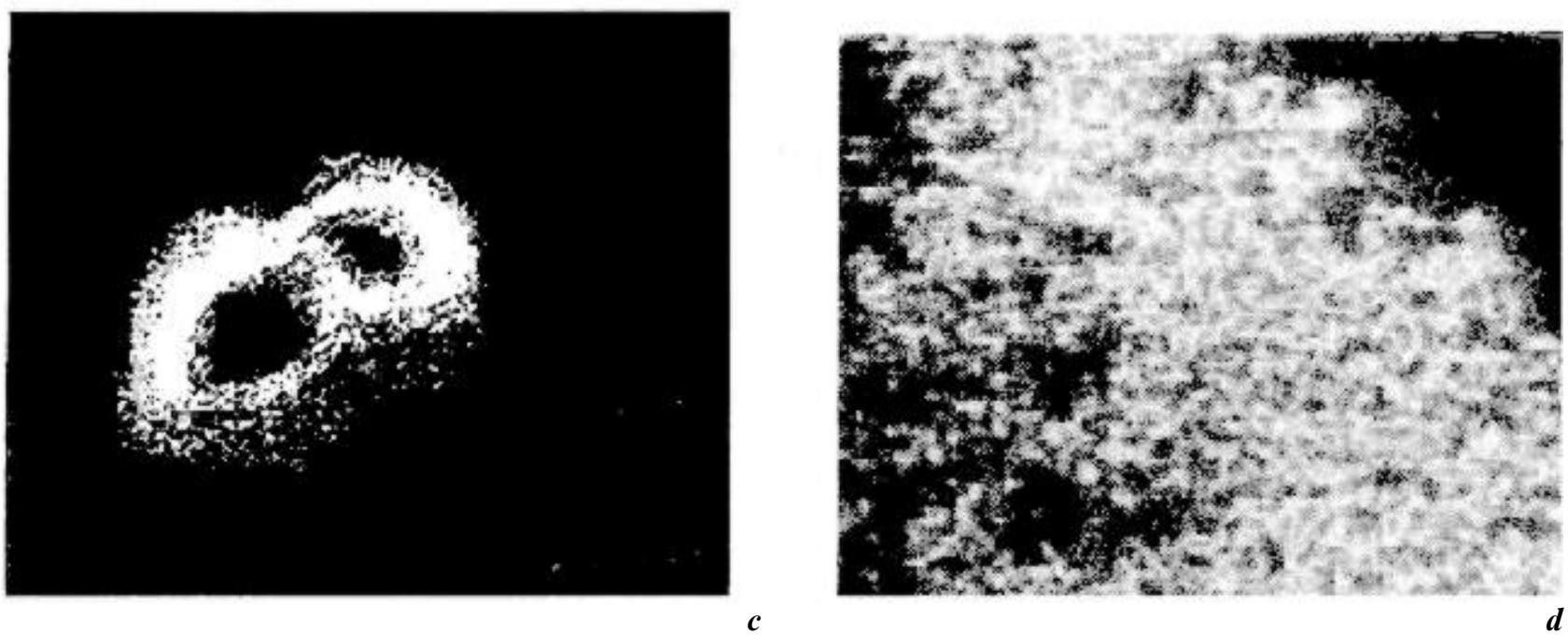

Fig. 5. Typical changes of patterns resulting from variation of $\alpha$ in EBE TFELS made in one and the same technological cycle with constant $d_{S}(\sim 580 \mathrm{~nm})$ and different $d_{i} . \alpha: 0.45(a) ; 0.41(b) ; 0.39(c) ; 0.37(d) . f=100 \mathrm{kHz}$.

tivator's effective range is the characteristic length of $A_{d}$, i.e. the distance traveled by the propagating charge in the time interval $T / 2$, where $T$ is the period of the applied voltage. This characteristic length depends on the mechanism of the charge spreading, which, in turn, depends on the surface morphology of the $\mathrm{ZnS}: \mathrm{Mn}$ film and the energy depth of filled electron interface states, on the one hand, and on the sample temperature, on the other hand. The activator's characteristic timescale $\left(\tau_{A}\right)$ depends on the characteristic timescales of both $A_{l}$ and $A_{d}$. The timescale $\left(\tau_{A l}\right)$ of the local activator is determined by the time that is necessary to recover the initial magnitude of the charge in a switched - on area after its decrease due to the lateral spreading of electrons. This time decreases as the driving frequency increases. The temperature affects $\tau_{A l}$ in two ways. It enhances the thermo-field generation of free electrons at given $V$, but it reduces the threshold field of this process and consequently the probability of both the impact multiplication of electrons and the formation of PSC. The distributed activator's timescale $\tau_{A d}$ is inversely proportional to the probability of the lateral spreading of the non-equilibrium charge, which is dependent on the particular mechanism of this process, i.e. on the factors mentioned above that determine the effective range of the activator .

The inhibitor may also be divided into a local $\left(I_{1}\right)$ and a distributed (Id) one. In addition, a global inhibitor $\left(I_{g 1}\right)$ should be considered. The first is due to the recombination of the charge carriers in the $\mathrm{ZnS}: \mathrm{Mn}$ film, which is a process with the NFB relative to the electron generation processes. The characteristic time of $I_{1}$ depends on the concentration of recombination centers, in particular of $V_{\mathrm{Zn}}$, as well as on the temperature and other factors affecting the number of free electrons. The role of the distributed inhibitor is the redistribution of the voltage between the $S$ layer and $I$ layers during the charge transfer 


\section{N.A. Vlasenko et al.: Self-organization patterns in electroluminescence of bistable...}

through the $S$ layer. The effective range of this inhibitor is characterized by $d_{i}$. Its characteristic timescale is determined by the time required to decrease the voltage drop across the $\mathrm{S}$ layer by an amount equal to the hysteresis loop width. Moreover, it depends on the $R C$ value, where $R$ is the resistance of the $S$ layer in the switched on state and $C$ is the capacitance of the $I$ layers. The global inhibitor is the voltage drop across the ITO electrode, which significantly increases with $f$ due to the increasing capacitive current. This resulting decrease of the voltage drop across the ISI structure could be compensated by the corresponding increase of the applied voltage. However, in this case a rather strong heating of the electrode and consequently of the whole sample that affects all processes dependent on temperature, should be taken into acount.

The dependence of the activator and the inhibitor on many factors ( the most important factor are: the concentration of intrinsic and impurity defects, the surface morphology of the $\mathrm{ZnS}: \mathrm{Mn}$ film, the relative thickness of the I and $\mathrm{S}$ layers, the temperature and the driving frequency) leads to the formation of a great variety of patterns in the emission of TFELS. The sensivity to both the sample properties and the driving conditions creates manifold opportunities to manipulate and control the patterns. However, the complexity of the interdependent processes in the films make difficult a mathematical modeling of the self-organization phenomenon in TFELS. Until now, only onedimensional processes responsible for the hysteresis of the $Q(V)$ characteristic have been modeled mathematically (see, e.g. [9, 23-26]). There are no publications of simulation results based on at least two-dimensional models which describe the formation of the patterns in the TFELS emission.

\section{References}

1. B.S. Kerner, V.V. Osipov. Autosolitons // Kluwer, Dordrecht, 1994.

2. J. Parisi, S.C. Muller, W. Zimmermann (Eds.). Nonlinear Physics of complex systems // Springer, Berlin, 1996.

3. F.H. Busse, S.C. Muller (Eds.) Evolution of spontaneous structures in dissipative continuous systems // Springer, 1998.

4. A.I. Beletskii, N.A. Vlasenko. Autowaves in the thin - film electroluminescent structures with intrinsic memory // Tech. Phys. Lett. 19(1), pp. 13-14 (1993).

5. A.I. Beletskii, N.A. Vlasenko. Flip-flop autowaves in the thinfilm electroluminescent structures // Tech. Phys. Lett. 19(12), pp. 798-799 (1993)

6. Ch. Goßen, F.-J. Niedernostheide, H.-G. Purwins. Pattern Formation of the Electroluminescence in AC ZnS:Mn Devices // in: Springer Proc. Phys., vol. 79, Ed. F.-J. Niedernostheide, Springer Verlag, Berlin / Heidelberg 1995, pp. 117-132.

7. H. Rufer, V. Marrello, A. Onton. Domain electroluminescence in ac thin-film devices // J Appl. Phys. 51, pp. 1163 1169 (1980).

8. A.I. Beletskii, N.A. Vlasenko, Hysteretic thin-film electroluminescent devices as dissipative autowave systems // in: Proc. 7th Intern. Workshop on Electroluminescence. Beijing, China, Oct. 10-12, 1994, pp. 415-419.

9. A.I. Beletskii. Physical processes in thin-film electroluminescent structures with inherent memory // Abstract of PhD Tesis, Institute of Semiconductor Physics, NAS of Ukraine, Kiev, 1995.

SQO, 7(1), 2004
10. A.I. Beletskii, L.I. Veligura, N.A. Vlasenko, Ya.F. Kononets. Effect of various factors on inherent memory of thin-film electroluminescent structures based on $\mathrm{ZnS}: \mathrm{Mn} / /$ in: Optoelectronika i poluprovodnikovaya technika. Kiev, Naukova Dumka, v. 24, 1992, pp. 99-108

11. N.A. Vlasenko, A.I. Beletskii, Z.L. Denisova, Ya. F. Kononets, L.I. Veligura. On causes of instability in beginning of aging of evaporated ZnS:Mn AC TFEL Devices // in: Inorganic and Organic Electroluminescence. Eds. P.H. Mauch, H.-E. Gumlich. Wissenschaft Technik Verlag, Berlin, 1996, pp. 267-270.

12. Ya. F. Kononets, R. Tornqvist, N.A. Vlasenko. On Physical Model of Aging Behaviour of Atomic Layer Epitaxy ZnS:Mn TFEL Devices // Ibid. pp. 259-262.

13. A.I. Beletskii, N.A. Vlasenko, L.I. Veligura. Frequency Dependence of Hysteresis of Characteristics of $\mathrm{ZnS}: \mathrm{Mn}$ TFEL Devices // Ibit. pp. 391-394.

14. S. Zuccaro, F.-J. Niedernostheide, B. Kukuk, M. Strych, H.-G. Purwins. Solitary current-density patterns in thin ZnS:Mn films // Phys. Rev. E 62, pp. $1284-1289$ (2000).

15. N.A. Vlasenko, A.I. Beletskii, Z.L. Denisova, L.I. Veligura, S. Zuccaro, F.-J. Niedernostheide, H.-G. Purwins. Energy levels of defects in electroluminescent $\mathrm{ZnS}: \mathrm{Mn}$ thin films exhibiting hysteresis and self - organized patterns. // $\mathrm{J}$. Cr . Growth, 214/215, pp. 944-949 (2000).

16. N.A. Vlasenko, M.M. Chumachkova, Z.L. Denisova, L.I. Veligura. On nature of centers responsible for inherent memory in $\mathrm{ZnS}: \mathrm{Mn}$ thin-film electroluminescent devices // $\mathrm{J}$. Cr. Growth, 216, pp. 249-255 (2000).

17. N.A. Vlasenko, Z.L. Denisova, Ya. F. Kononets, L.I. Veligura, I. A. Gumenyuk, S. Zuccaro, F.-J. Niedernostheide, H.-G. Purwins. Effect of Insulator Layer Properties in ZnS:Mn AC TFEL Structures on Self-Organized Patterns. // in: Proc. 10th Intern. Workshop on Inorganic and Organic Electroluminescence, Hamamatsu, Japan, Dec. 4-7, 2000, pp. 85-88.

18. N.A. Vlasenko, H.-G. Purwins, Ya.F. Kononets, F.-J. Niedernostheide, L.I. Veligura, S. Zuccaro, I.A. Gumenyuk. On Role of Temperature in Formation of Self-Organized Patterns in Emission of Bistable ZnS:Mn TFEL Structures // in: Proc. $11^{\text {th }}$ Intern. Workshop on Inorganic and Organic Electroluminescence. Ghent, Belgium Sept. 23-26, 2002, pp. 367-370.

19. N.A. Vlasenko, H.-G. Purwins, V.M. Popov, I.A. Gumenyuk, A.S. Klimenko, Ya.F. Kononets, F.-J. Niedernostheide, L.I. Veligura, S. Zuccaro. Temperature effects on Self - Organized Patterns and Characteristics of Bistable ZnS:Mn ThinFilm Structures // Phys. Stat. Sol. (a) 194, pp. 237-243 (2002).

20. T. Suntola, J. Antson, A. Pakkola, S. Lindfors. Atomic Layer Epitaxy for Producting EL-Thin Films // in SID 1980 Dig. Tech. Paper, vol. XI, pp. 108-109 (1980).

21. D. Theis. Selected Analytical Tools Yield a Better Insight into Electroluminescent Thin Films // Phys. Stat. Sol. (a) 81, pp. 647-655 (1984).

22. N.A. Vlasenko, L.I. Veligura, Z.L. Denisova, Ya. F. Kononets, I.A. Gumenyuk, H.-G. Purwins, F.-J. Niedernostheide, S. Zuccaro. Spatially inhomogeneous states in electroluminescent bistable thin - film structures based on $\mathrm{ZnS}: \mathrm{Mn} / / A b$ stracts of $1^{\text {st }}$ Ukrainian Scienc. Conf. on Semicond. Phys. Odessa, Ukraine, Sept. 10-14, 2002, v.1, pp. 94-95.

23. W.E. Howard, O. Sahni, P.M. Alt. A simple model for the hysteretic behavior of $\mathrm{ZnS}: \mathrm{Mn}$ thin-film electroluminescent devices // J. Appl. Phys. 53, pp. 639-647 (1982).

24. J.M. Jarem. V.P. Singh. A Computationally Simple Model for Hysteretic Thin Film Electroluminescent Devices // IEEE Trans. Electron Devices. 35, pp. 1834-1841 (1988).

25. K.A. Neits. Simple Model for the Hysteretic Behavior of Thin-Film Electroluminescent Devices // IEEE Trans. Electron Devices, 38, pp.2604-2611 (1991).

26. S.Zuccaro, Th. Raker, F.-J. Niedernostheide, T. Kuhn, H.-G. Purwins. Physical processes in thin-film electroluminescent structures based on $\mathrm{ZnS}$ :Mn showing self-organized patterns // Chaos Solitons Fractals, 17, p.p. 321-236 (2003). 